\title{
Osteological correlates for quadrupedality in ornithischian dinosaurs
}

\author{
Susannah C.R. Maidment and Paul M. Barrett
}

Acta Palaeontologica Polonica 59 (1), 2014: 53-70 doi: http://dx.doi.org/10.4202/app.2012.0065

The evolution of quadrupedality from bipedal ancestors is an exceptionally rare transition in tetrapod evolution, but it has occurred several times within the herbivorous dinosaur clade Ornithischia. Stegosauria, Ankylosauria, and Ceratopsidae are all uncontroversially quadrupedal, while basal ornithischians and basal ornithopods are uncontroversially bipedal. However, stance in iguanodontian ornithopods, including the hadrosaurs, and in non-ceratopsid ceratopsians is debated because robust osteological correlates of quadrupedality have not been identified. We examine a suite of characteristics that have been previously proposed as osteological correlates for bipedality or quadrupedality in dinosaurs. These include both discrete anatomical features, which we assess as correlates for quadrupedality using character optimization onto a composite cladogram, and proportional ratios, which we assess as correlates by reconstructing nodal ancestral states using squared-change parsimony, followed by optimization. We also examine the correlation of these features with body size. An anterolateral process on the proximal ulna, hoof-shaped manual unguals, a transversely broadened ilium, a reduced fourth trochanter and a femur longer than the tibia are found to be robust correlates of quadrupedality in ornithischian dinosaurs. Along the ceratopsid "stem" lineage, quadrupedal characters were acquired in a stepwise fashion, with forelimb characters developing prior to changes in the hind limb. In contrast, iguanodontid ornithopods display a mosaic of character states, indicating varying degrees of facultative quadrupedality that probably arose for a variety of different reasons. Hadrosaurs are found to possess all character states associated with quadrupedality and were probably predominantly quadrupedal. In general, quadrupedal ornithischians do not appear to have been constrained by their bipedal ancestry to a particular order of character acquisition.

Key words: Dinosauria, Ornithischia, osteological correlate, quadrupedality.

Susannah C. R. Maidment [s.maidment@imperial.ac.uk], Department of Earth Sciences, The Natural History Museum, Cromwell Road, London, SW7 5BD, United Kingdom; current address: Department of Earth Science and Engineering, Imperial College, South Kensington Campus, London SW7 2AZ, UK; Paul M. Barrett [p.barrett@nhm.ac.uk], Department of Earth Sciences, The Natural History Museum, Cromwell Road, London, SW7 5BD, UK. 
This is an open-access article distributed under the terms of the Creative Commons

Attribution License (for details please see creativecommons.org), which permits unrestricted use, distribution, and reproduction in any medium, provided the original author and source are credited.

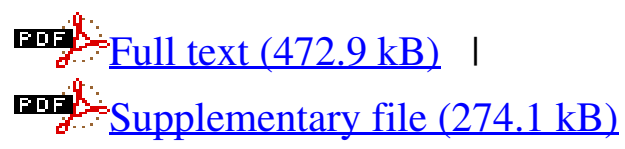

